\title{
CONFORMAL SUBFOLIATIONS OF PRESCRIBED GEODESIC CURVATURE
}

\author{
PAUL BAIRD and JEAN-MARIE BUREL**
}

\begin{abstract}
Given a 2-dimensional conformal foliation $\mathscr{F}$ of a Riemannian manifold $M$, the problem of finding a 1-dimensional subfoliation $\mathscr{G}$, conformal in $M$, whose leaves have prescribed geodesic curvature in the leaves of $\mathscr{F}$ is equivalent to a Pfaff differential system on a circle bundle over $M$. We study such pairs of foliations on a 3- and 4-manifold.
\end{abstract}

\section{Introduction}

Let $M^{m}$ be a smooth $m$-dimensional manifold; then an ordered pair of foliations $(\mathscr{F}, \mathscr{G})$ is called a flag if the leaves of $\mathscr{F}$ contain those of $\mathscr{G}$. The notion was introduced by Feigin [6]; they were subsequently studied by Cordero and Masa [5], who called such a pair a subfoliation. We will retain the term flag for such a pair and say that $\mathscr{G}$ is a subfoliation of $\mathscr{F}$; in this case we will write $\mathscr{G} \subset \mathscr{F}$. All objects are smooth unless otherwise stated.

Let $\mathscr{F}$ be a foliation of $M$. Given $x_{0} \in M$, there is a neighbourhood $U$ of $x_{0}$ and a diffeomorphism (called a foliated chart) $\psi: U \rightarrow U_{1} \times U_{2} \subset \mathbf{R}^{k} \times \mathbf{R}^{n}$ such that each connected component of the intersection of any leaf with $U$ (called a plaque of $\mathscr{F}$ in $U$ ) is given by $\psi^{-1}\left(U_{1} \times y\right)$ for some $y \in U_{2}$. The corresponding submersion $\pi_{2} \circ \psi: U \rightarrow U_{2} \subset \mathbf{R}^{n}$, where $\pi_{2}: \mathbf{R}^{k} \times \mathbf{R}^{n} \rightarrow \mathbf{R}^{n}$ is the projection onto the second factor, is called a distinguished submersion; see, for example [7]. Let $T \mathscr{F}$ denote the associated tangent distribution to $\mathscr{F}$, so that at each $x \in M, T_{x} \mathscr{F} \subset T_{x} M$ gives the tangent space to the leaf of $\mathscr{F}$ passing through $x$. Let $N \mathscr{F}$ denote the normal distribution $(T \mathscr{F})^{\perp}$. Then at each $x \in M, T_{x} M=T_{x} \mathscr{F} \oplus N_{x} \mathscr{F}$ gives an orthogonal decomposition of the tangent bundle. In general, if $E$ is a vector bundle over $M$, we let $\Gamma(E)$ denote the space of (smooth) sections of $E$.

A foliation $\mathscr{F}$ on a Riemannian manifold $(M, g)$ is said to be conformal (or transversally conformal) if parallel transport of normal vectors along the

\footnotetext{
* The second named author is partially supported by The European Contract HPRN-CT-200000101.

Received April 4, 2002.
} 
leaves is conformal. Precisely,

$$
\left(\mathscr{L}_{V} g\right)(X, Y)=a_{x}(V) g(X, Y),
$$

for all $V \in \Gamma(T \mathscr{F}), X, Y \in N_{x} \mathscr{F}, x \in M$, where $\mathscr{L}_{V}$ denotes Lie derivation and $a_{x}(V)$ depends only on $x$ and $V$. Any codimension 1-foliation is conformal. A special case of a conformal foliation is a Riemannian foliation, which corresponds to $a$ being identically zero. We call $a \in \Gamma\left(T^{*} \mathscr{F}\right)$ the $d i$ vergence associated to the conformal foliation. Indeed, if $\mathscr{F}$ is an oriented 1-dimensional conformal foliation and $V$ is a choice of unit tangent vector field, then a simple calculation shows that $a(V)=2 \operatorname{div} V /(m-1)$.

The property of a foliation being conformal has an interpretation in terms of distinguished submersions as follows. A map $\varphi:(M, g) \rightarrow(N, h)$ between Riemannian manifolds is said to be semi-conformal if, for each $x \in M$ where $\mathrm{d} \varphi_{x} \neq 0$, there exists a number $\lambda(x)>0$ such that $\varphi^{*} h(X, Y)=$ $\lambda(x)^{2} g(X, Y)$, for all $X, Y \in\left(\operatorname{ker} \varphi_{x}\right)^{\perp}$. Setting $\lambda(x)=0$ at points where $\mathrm{d} \varphi_{x}=0$ defines a continuous function $\lambda: M \rightarrow \mathrm{R}$ called the dilation of $\varphi$. The square $\lambda^{2}=|\mathrm{d} \varphi|^{2}$ is smooth on $M$. Then $\mathscr{F}$ is a conformal foliation if and only if for each distinguished submersion $\psi: U \subset M \rightarrow \mathrm{R}^{n}$, there is a Riemannian structure defined on $\mathbf{R}^{n}$ with respect to which $\psi$ becomes a semi-conformal submersion (see [10]). The relation between the divergence $a$ and the dilation $\lambda$ is given by $a(V)=-2 V(\ln \lambda)$. We will call the dilation $\lambda$ of a semi-conformal distinguished submersion a local dilation for the conformal foliation $\mathscr{F}$.

If $\mathscr{F}$ is a 2-dimensional oriented foliation of a Riemannian manifold $M^{m}$, then any oriented 1-dimensional subfoliation $\mathscr{G} \subset \mathscr{F}$ determines a function $\kappa: M^{m} \rightarrow \mathrm{R}$ as follows. At each point $x \in M^{m}$ we consider the leaves $G$ and $F$ of $\mathscr{G}$ and $\mathscr{F}$, respectively, which pass through $x$. Then we define $\kappa(x)$ to be the geodesic curvature of $G$ in $F$ at $x$. We shall call $\kappa$ the geodesic curvature of the foliation $\mathscr{G}$ in the foliation $\mathscr{F}$. If $\mathscr{F}$ is conformal, we show that the problem of finding a subfoliation $\mathscr{G}$ conformal in $M^{m}$ with prescribed geodesic curvature $\kappa$ is equivalent to solving a Pfaff differential system. In dimension 3 when $M^{3}$ has constant curvature, we characterise those foliations $\mathscr{F}$ together with the associated admissible functions $\kappa$ for which this system is completely integrable. Geometrically this means that for an admissible function $\kappa$, at any point $x_{0}$ of $M$ and unit vector $V$ at $x_{0}$ tangent to $\mathscr{F}$, there is precisely one conformal subfoliation with direction $V$ at $x_{0}$.

Another case of special interest is when $M$ has dimension 4 and $\mathscr{F}$ is a 2-dimensional minimal conformal foliation. Now adapted almost Hermitian structures will play an important role. By working on a dense subset of $S^{4}$, we describe examples of minimal 2-dimensional conformal foliations which admit 
a 1-dimensional conformal subfoliation (Example 4.9). This gives examples of almost Hermitian structures with integrable Lee form. We construct a family of 2-dimensional foliations on $S^{3} \times S^{1}$ with corresponding Pfaff differential system completely integrable.

The authors express their thanks to Frédéric Helein for pointing out the importance of Pfaff differential systems in this study.

\section{The associated differential system}

Let $\mathscr{F}$ be an oriented 2-dimensional conformal foliation of a Riemannian manifold $M^{m}$ of dimension $m \geq 3$. Let $\mathscr{G}$ be an arbitrary 1-dimensional oriented subfoliation of $\mathscr{F}$. Let $\left\{e_{1}, e_{2}, \ldots, e_{m}\right\}$ be a local orthonormal frame defined on an open set $U \subset M$ such that $\left\{e_{1}, e_{2}\right\}$ spans $T \mathscr{F}$ and let $\left\{\omega^{1}, \ldots, \omega^{m}\right\}$ be the dual frame. Then the connection matrix is the matrix of 1 -forms $\left\{\omega^{i j}\right\}$ determined by

$$
\nabla e_{i}=\omega^{i j} e_{j} \quad \text { (summing over repeated indices) }
$$

The structure equations (cf. [9]) are given by

$$
\left\{\begin{aligned}
\mathrm{d} \omega^{i} & =\omega^{i j} \wedge \omega^{j} \\
\mathrm{~d} \omega^{i j} & =\omega^{i k} \wedge \omega^{k j}+\frac{1}{2} R_{i j k l} \omega^{k} \wedge \omega^{l}
\end{aligned}\right.
$$

where the curvature components $R_{i j k l}$ are determined by the expression

$$
R_{i j k l}=\left\langle R\left(e_{i}, e_{j}\right) e_{k}, e_{l}\right\rangle=\left\langle\nabla_{e_{i}} \nabla_{e_{j}} e_{k}-\nabla_{e_{j}} \nabla_{e_{i}} e_{k}-\nabla_{\left[e_{i}, e_{j}\right]} e_{k}, e_{l}\right\rangle .
$$

Let $V$ be a unit tangent vector field to $\mathscr{G}$ on $U$. Then $V$ is given by

$$
V=\cos \alpha e_{1}+\sin \alpha e_{2},
$$

for some function $\alpha: U \rightarrow \mathrm{R}$. Although $\alpha$ is only defined on the domain $U$ of our orthonormal frame, the 1 -form $\mu=\mathrm{d} \alpha+\omega^{12}$ is well-defined everywhere. Write $J^{\mathscr{F}}$ for rotation by $+\pi / 2$ in $T \mathscr{F}$. The following lemma is elementary in the theory of surfaces.

Lemma 2.1. The 1-form $\mu=\mathrm{d} \alpha+\omega^{12}$ is well-defined, independently of the frame $\left\{e_{1}, \ldots, e_{m}\right\}$ and extends to a well-defined 1-form on $M$. The quantity $\kappa_{g}(V)=\mu(V)=\left\langle J^{\mathscr{F}} V, \nabla_{V} V\right\rangle$ measures the geodesic curvature of the integral curves of $V$.

We now study conditions that ensure $\mathscr{G}$ is a conformal foliation in $M^{m}$. Let $\theta$ be the dual form to $J^{\mathscr{F}} V$, so that $\theta(X)=\left\langle J^{\mathscr{F}} V, X\right\rangle$ for all $X \in T_{x} M$, $x \in M$. 
Lemma 2.2. The subfoliation $\mathscr{G}$ of $\mathscr{F}$ is conformal on $U$ if and only if

$$
2 \mathscr{L}_{V} \theta-a(V) \theta=0,
$$

where $a$ is the divergence associated to $\mathscr{F}$.

Proof. By definition $\mathscr{G}$ is a conformal foliation if and only if

$$
\left(\mathscr{L}_{V} g\right)(X, Y)=a(V) g(X, Y),
$$

for all $X, Y \in \Gamma(N \mathscr{G})$. Since $\mathscr{F}$ is conformal by hypothesis, this condition is automatically satisfied if $X, Y$ are normal to $\mathscr{F}$. The remaining cases: $X=$ $Y=J^{\mathscr{F}} V$ and $X=J^{\mathscr{F}} V, Y \in \Gamma(N \mathscr{F})$ give the equivalence between equations (3) and (2).

In terms of the local frame the above condition can be expressed as follows. We employ the musical isomorphisms b $: T M \rightarrow T^{*} M$ and $\sharp: T^{*} M \rightarrow T M$ characterized by $X^{\mathrm{b}}(Y)=g(X, Y)$ and $g\left(\omega^{\sharp}, X\right)=\omega(X)$, respectively, for all $X, Y \in T M, \omega \in T^{*} M$.

Corollary 2.3. The subfoliation $\mathscr{G}$ of $\mathscr{F}$ is conformal on $U$ if and only if there exists a function $\kappa: U \rightarrow \mathrm{R}$ such that

$$
\mathrm{d} \alpha+\omega^{12}+\left(\nabla_{V} \theta\right) \circ \mathcal{N}-\frac{a(V)}{2} \theta+\kappa V^{b}=0,
$$

where $\mathcal{N}$ is orthogonal projection onto the normal space $N_{x} \mathscr{F}$ at each $x \in U$.

REMARK 2.4. (i) The function $\kappa$ arises, since the left-hand side of (2) can be written $2 \mathscr{L}_{V} \theta-a(V) \theta=2 \mathrm{~d}(\lambda \theta)(V, \cdot)$, for any local dilation $\lambda$, which automatically vanishes on $V$. In order to establish that $\mathscr{G}$ is conformal, we do not need to know a priori the value of $\kappa$; it simply suffices to know that there is some function $\kappa$ such that (4) is satisfied. If such exists, then it corresponds to the geodesic curvature of $\mathscr{G}$ in $\mathscr{F}$.

(ii) The term $\left(\nabla_{V} \theta\right) \circ \mathscr{N}$ depends only on $\alpha$ and not its derivatives at each point. This will be important in our considerations below.

Consider the circle bundle $T^{1} \mathscr{F} \rightarrow M^{m}$ whose fibre at each point $x$ is given by the circle of unit vectors in $T_{x} \mathscr{F}$. Then on $U$, we can identify $T^{1} \mathscr{F}$ with the product $U \times S^{1}$ by identifying $V=\cos y e_{1}+\sin y e_{2} \in T_{x}^{1} \mathscr{F}$ with the point $\left(x, e^{\mathrm{i} y}\right) \in U \times S^{1}$. For a given function $\kappa: U \rightarrow \mathrm{R}$, consider the 1 -form on $U \times \mathrm{R}$ defined by

$$
\Omega_{\kappa} \equiv \mathrm{d} y+\omega^{12}+\left(\nabla_{V} \theta\right) \circ \mathcal{N}-\frac{a(V)}{2} \theta+\kappa V^{\mathrm{b}} .
$$


Note that this is indeed a 1-form on $U \times \mathrm{R}$ by Remark 2.4(ii) above, so we now regard $V$ and $\theta$ as depending on $y \in \mathbf{R}$. Our first observation is that the equation $\Omega_{\kappa}=0$ represents a Pfaff differential system on $U \times \mathrm{R}$.

Recall that a Pfaff differential system on a manifold $P$ is a set of $l$ equations $\left\{\Omega^{i}=0\right\}, i=1, \ldots l$, where each $\Omega^{i}$ is a differential 1 -form [1]. Provided the $\Omega^{i}$ are independent, we say that the system has rank $l$.

Let $W$ be a submanifold of $P$ and let $\iota: W \hookrightarrow P$ be the inclusion map, then $W$ is said to be an integral manifold of the system if $\iota^{*} \Omega^{i}=0$ for all $i$. The system is said to be completely integrable is through each point $p_{0} \in P$, there is exactly one maximal integral submanifold of dimension $\operatorname{dim} P-l$. Equivalently ([1], 3.17, 3.18)

$$
\mathrm{d} \Omega^{i} \wedge \Omega^{1} \wedge \cdots \wedge \Omega^{l}=0 \quad 1 \leq i \leq l .
$$

In our situation, we set $P=U \times \mathrm{R}$. For given $\kappa$, the equation

$$
\Omega_{\kappa} \equiv \mathrm{d} y+\omega^{12}+\left(\nabla_{V} \theta\right) \circ \mathcal{N}-\frac{a(V)}{2} \theta+\kappa V^{b}=0
$$

defines a Pfaff differential system of rank 1 . The complete integrablity condition now becomes

$$
\mathrm{d} \Omega_{\kappa} \wedge \Omega_{\kappa}=0
$$

An integrable submanifold of dimension $m(=\operatorname{dim} M)$ which is transverse to the fibres of the bundle $U \times \mathrm{R} \rightarrow U$ locally determines a function $\alpha: U \rightarrow \mathrm{R}$ which in turn determines a vector field $V$ whose integral curves are the leaves of a conformal subfoliation $\mathscr{G} \subset \mathscr{F}$. The objects given in order to determine a well-defined Pfaff differential system are the manifold $M$, the foliation $\mathscr{F}$ and the function $\kappa: M \rightarrow \mathrm{R}$. We pose the following fundamental problems:

Problem 1. For which 2-dimension oriented conformal foliations $\mathscr{F}$ does there exist a function $\kappa: M \rightarrow \mathrm{R}$ such that the corresponding Pfaff differential system (6) is completely integrable?

Problem 2. Given such a foliation $\mathscr{F}$, what are the admissible functions $\kappa$ which render the system completely integrable?

On $U \times \mathrm{R}$, consider a more general Pfaff differential system of the form

$$
\Omega \equiv \mathrm{d} y-a_{i}(x, y) \omega^{i}=0
$$

where $\left\{\omega^{i}\right\}$ are 1-forms dual to an orthonormal frame $\left\{e_{i}\right\}$ defined on an open set $U \subset M$ and $(x, y) \in U \times \mathrm{R}$. 
We say that a function $\alpha: U \rightarrow \mathrm{R}$ provides a solution to (8) if

$$
\mathrm{d} \alpha-a_{i}(x, \alpha(x)) \omega^{i}=0 .
$$

Proposition 2.5. (i) If $\alpha: U \rightarrow \mathrm{R}$ provides a solution to the system (8), then

$$
\left\{e_{i}\left(a_{k}\right)+a_{i} \frac{\partial a_{k}}{\partial y}\right\}(x, \alpha(x)) \omega^{i} \wedge \omega^{k}+a_{i}(x, \alpha(x)) \mathrm{d} \omega^{i}=0,
$$

for all $x \in U$. (Here and henceforth, we apply the Einstein summation convention and sum over repeated indices.)

(ii) The system (8) is completely integrable if and only if

$$
\left\{e_{i}\left(a_{k}\right)+a_{i} \frac{\partial a_{k}}{\partial y}\right\}(x, y) \omega^{i} \wedge \omega^{k}+a_{i}(x, y) \mathrm{d} \omega^{i}=0,
$$

for all $(x, y) \in U \times \mathrm{R}$.

Proof. (i) On taking the exterior derivative of both sides of the equation

$$
\mathrm{d} \alpha=a_{k}(x, \alpha) \omega^{k}
$$

we obtain

$$
\begin{aligned}
0 & =e_{i}\left(a_{k}\right) \omega^{i} \wedge \omega^{k}+\frac{\partial a_{k}}{\partial y} \mathrm{~d} \alpha \wedge \omega^{k}+a_{k} \mathrm{~d} \omega^{k} \\
& =\left(e_{i}\left(a_{k}\right)+a_{i} \frac{\partial a_{k}}{\partial y}\right) \omega^{i} \wedge \omega^{k}+a_{i} \mathrm{~d} \omega^{i}
\end{aligned}
$$

giving the necessary condition (9).

(ii) Complete integrablity is equivalent to the vanishing of $\Omega \wedge \mathrm{d} \Omega$ viewed as a form on $U \times \mathrm{R}$. Now

$$
\begin{gathered}
\Omega \wedge \mathrm{d} \Omega=-\mathrm{d} y \wedge\left\{\left(e_{i}\left(a_{k}\right)+a_{i} \frac{\partial a_{k}}{\partial y}\right) \omega^{i} \wedge \omega^{k}+a_{i} \mathrm{~d} \omega^{i}\right\} \\
+a_{j} \omega^{j} \wedge\left\{e_{i}\left(a_{k}\right) \omega^{i} \wedge \omega^{k}+a_{i} \mathrm{~d} \omega^{i}\right\}
\end{gathered}
$$

The term involving $\mathrm{d} y$ must vanish independently, i.e.

$$
\begin{array}{r}
\left(e_{i}\left(a_{k}\right)+a_{i} \frac{\partial a_{k}}{\partial y}\right) \omega^{i} \wedge \omega^{k}+a_{i} \mathrm{~d} \omega^{i}=0 \\
a_{j} \omega^{j} \wedge\left\{e_{i}\left(a_{k}\right) \omega^{i} \wedge \omega^{k}+a_{i} \mathrm{~d} \omega^{i}\right\}=0
\end{array}
$$


From (11), $e_{i}\left(a_{k}\right) \omega^{i} \wedge \omega^{k}+a_{i} \mathrm{~d} \omega^{i}=-a_{i} \frac{\partial a_{k}}{\partial y} \omega^{i} \wedge \omega^{k}$ and (12) becomes

$$
a_{i} a_{j} \frac{\partial a_{k}}{\partial y} \omega^{i} \wedge \omega^{j} \wedge \omega^{k}=0 .
$$

But the product $a_{i} a_{j}$ is symmetric in the indices $i, j$ whereas $\omega^{i} \wedge \omega^{j}$ is antisymmetric, so (13) is automatically satisfied and the only independent condition is (11).

We apply the above proposition to the system (6).

Corollary 2.6. Let $\kappa: U \rightarrow \mathrm{R}$ be a smooth function and consider the Pfaff differential system (6).

(i) If $\alpha: U \rightarrow \mathrm{R}$ determines a solution to the system, so that the subfoliation $\mathscr{G} \subset \mathscr{F}$ is conformal on $U$, then the equations (9) are necessary conditions that must be satisfied.

(ii) The system is completely integrable on $U$ if and only if (10) are satisfied for all $(x, y) \in U \times \mathbf{R}$. In this case, for each $x_{0} \in U$ and each direction $V \in T_{x_{0}}^{1} \mathscr{F}$ at $x_{0}$, there is precisely one conformal subfoliation $\mathscr{G}$ of $\mathscr{F}$ defined in a neighbourhood of $x_{0}$ with direction $V$ at $x_{0}$.

One geometric condition that must be satisfied in order that $\mathscr{G}$ be a conformal subfoliation $\mathscr{G}$ is given by the following.

Proposition 2.7. Let $\mathscr{F}$ be an oriented conformal foliation of dimension 2 of a Riemannian manifold $(M, g)$ with associated divergence $a \in \Gamma\left(T^{*} \mathscr{F}\right)$ and let $\mathscr{G}$ be a 1-dimensional subfoliation. Then if $\mathscr{G}$ is conformal,

$$
K+\frac{V(a(V))}{2}+\frac{a(V)^{2}}{4}+W(\kappa)+\kappa^{2}=0
$$

where $K$ is the Gauss curvature of the leaves of $\mathscr{F}$ and $\kappa$ is the geodesic curvature of $\mathscr{G}$ in $\mathscr{F}$.

Proof. We apply part (i) of Corollary 2.6 by evaluating the left-hand-side of (9) with $\Omega=\Omega_{\kappa}$ on the pair $(V, W)$. This is equivalent to evaluating the exterior derivative of the left-hand-side of $(4)$ on $(V, W)$. Firstly

$$
\begin{aligned}
\mathrm{d} \omega^{12}(V, W) & =\mathrm{d} \omega^{12}\left(e_{1}, e_{2}\right)=\left(\omega^{1 k} \wedge \omega^{k 2}\right)\left(e_{1}, e_{2}\right)+R_{1212} \\
& =-\omega^{1 k}\left(e_{1}\right) \omega^{2 k}\left(e_{2}\right)+\sum_{k} \omega^{1 k}\left(e_{2}\right)^{2}+R_{1212}
\end{aligned}
$$

by the integrability of the leaves of $\mathscr{F}$. Now the Gauss equation for the leaves of $\mathscr{F}$ gives $R_{1212}=R_{1212}^{\mathscr{F}}-\sum_{k} \omega^{1 k}\left(e_{2}\right)^{2}+\sum_{k} \omega^{1 k}\left(e_{1}\right) \omega^{2 k}\left(e_{2}\right)$ where $R^{\mathscr{F}}$ 
denotes the curvature tensor of the leaves of $\mathscr{F}$, so that $R_{1212}^{\mathscr{F}}=-K$. Therefore $\mathrm{d} \omega^{12}(V, W)=-K$.

For the other terms we have, $\mathrm{d}\left(\mathcal{N} \nabla_{V} W\right)^{\mathrm{b}}(V, W)=-\left(\mathcal{N} \nabla_{V} W\right)^{\mathrm{b}}(\mathcal{N}[V, W])$ which vanishes by the integrability of $\mathscr{F}$. On the other hand $\mathrm{d}(a(V) \theta)(V, W)=V(a(V)) \theta(W)+a(V) \mathrm{d} \theta(V, W)=V(a(V))+\frac{1}{2} a(V)^{2}$.

Finally, $\mathrm{d}\left(\kappa V^{\mathrm{b}}\right)(V, W)=-W(\kappa)+\kappa\left(\mathrm{d} V^{\mathrm{b}}\right)(V, W)=-W(\kappa)-\kappa g(V,[V, W])$ $=-W(\kappa)-\kappa^{2}$.

Corollary 2.8. Let $(\mathscr{F}, \mathscr{G})$ be a conformal flag of dimension $(2,1)$. If $\mathscr{F}$ is Riemannian with compact leaves satisfying $K \equiv 0$, then $\kappa \equiv 0$, i.e. the leaves of $\mathscr{G}$ are (parallel and) geodesic in $\mathscr{F}$.

Proof. The fundamental equation (14) becomes

$$
\mathrm{d} \kappa(W)+\kappa^{2}=0 .
$$

At a critical point of $\kappa$ we have $\mathrm{d} \kappa=0$ in which case $\kappa=0$ and $\kappa$ is identically zero.

\section{Complete integrability in dimension three}

In this section, we classify those flags $(\mathscr{F}, \mathscr{G})$ of conformal foliations on a 3 manifold of constant sectional curvature, for which the differential system (6) is completely integrable. Recall that a codimension 1 foliation is automatically conformal.

THEOREM 3.1. Let $\mathscr{F}$ be an oriented 2-dimensional (conformal) foliation of an open subset $U$ of an oriented manifold $M^{3}$ of constant sectional curvature. Let $\kappa: U \rightarrow \mathrm{R}$ prescribe the geodesic curvature. If the corresponding Pfaff differential system (6) is completely integrable then the foliation $\mathscr{F}$ is one of the following types:

(i) a family of parallel hypersurfaces of constant mean curvatures;

(ii) a family of totally geodesic surfaces which extend to a common geodesic in $M$.

Conversely, if $\mathscr{F}$ is one of these types then there exist admissible functions $\kappa: U \rightarrow \mathrm{R}$ such that the Pfaff differential system is completely integrable. Given such a function there exists precisely one conformal subfoliation with prescribed direction at a given point. The different cases together with their associated admissible functions are described in $(a)-(d)$ below.

REMARK 3.2. A family given by (i) is called an isoparametric family of hypersurfaces. The property of being parallel and of constant mean curvatures 
means that the principal curvatures are also constant along each hypersurface. In $\mathrm{R}^{3}$, such a family consists of one of the following: (a) parallel planes; (b) co-axial cylinders; (c) concentric spheres [4].

We first establish the following lemma.

LEMMA 3.3. Let $\mathscr{F}$ be as in the statement of the theorem with normal vector field $e_{3}$ geodesic. Let $\kappa: U \rightarrow \mathrm{R}$ be given. If the corresponding Pfaff differential system (6) is completely integrable then the foliation $\mathscr{F}$ is by parallel surfaces of constant mean curvatures.

Proof. Let $k_{1}, k_{2}$ be the principal curvatures of the leaves of $\mathscr{F}$ in $M$ and suppose that $e_{1}, e_{2}$ are chosen to lie in the corresponding principal directions on the open subset $U \subset M$. First note that the normal vector field to $\mathscr{F}$ given by $e_{3}$ is geodesic if and only if the divergence $a$ is identically zero. With the hypotheses of the lemma, the equations (10) for complete integrability become

(i) $k_{1} k_{2}+\mathrm{d} \kappa(W)+\kappa^{2}=0$;

(ii) $\sin y \cos y V\left(k_{1}-k_{2}\right)+e_{3}(\kappa)-\kappa\left(k_{1} \cos ^{2} y+k_{2} \sin ^{2} y\right)=0$;

(iii) $\sin y \cos y W\left(k_{1}-k_{2}\right)+2 \kappa \sin y \cos y\left(k_{1}-k_{2}\right)=0$.

for all $(x, y) \in U \times \mathrm{R}$. Since $\sin y \cos y, \cos ^{2} y$ are independent functions, from (ii) we have

$$
V\left(k_{1}-k_{2}\right)=0, \quad e_{3}(\kappa)-k_{2} \kappa=0, \quad \kappa\left(k_{1}-k_{2}\right)=0 .
$$

Thus, either $k_{1}-k_{2}=0$ and the leaves of $\mathscr{F}$ are umbilic and so are parts of planes or spheres, or $\kappa=0$. In this latter case, from (i) $k_{1} k_{2}=0$ and from (iii) $W\left(k_{1}-k_{2}\right)=0$. Then one of the principal curvatures, say $k_{2}$ vanishes and the other is constant along the leaves since $V\left(k_{1}\right)=W\left(k_{1}\right)=0$.

Proof of Theorem 3.1. From equations (10), we deduce the following set of equations:

$$
\begin{aligned}
k_{2}\left\langle e_{1}, \nabla_{e_{3}} e_{3}\right\rangle=k_{1}\left\langle e_{2}, \nabla_{e_{3}} e_{3}\right\rangle & =0 \\
W\left(k_{1}-k_{2}\right)+\frac{1}{2}\left(k_{1}-k_{2}\right) a(W)+2 \kappa\left(k_{1}-k_{2}\right) & =0 \\
\left(k_{1}-k_{2}\right) a(V) & =0 \\
-e_{3}(a(V))+k_{2} a(V) & =0
\end{aligned}
$$

Then either $\nabla_{e_{3}} e_{3}=0$ and by Lemma 3.3, $\mathscr{F}$ is one of the types given by (i) of the theorem, or $\nabla_{e_{3}} e_{3} \neq 0$ on an open set $W \subset U$. But then one of $k_{1}, k_{2}$ must vanish. If they are not both zero, then $a(V)=0$, contradicting our assumption that $\nabla_{e_{3}} e_{3} \neq 0$. Thus we must have both $k_{1}, k_{2}$ vanishing and the leaves of $\mathscr{F}$ 
are totally geodesic surfaces. That each leaf extends to a common geodesic is established in (d) below.

We establish the converse as well as completing the proof of part (ii) of the theorem. We must show that for each of the given types, there are admissible functions $\kappa$ such that the corresponding Pfaff differential system is completely integrable. By lifting to a covering if necessary, we may suppose that $M^{3}$ is a simply connected space-form. For ease of exposition we suppose that $M^{3}=\mathrm{R}^{3}$; the spherical and hyperbolic cases being similar. We consider each case in turn.

(a) Parallel planes $\left(k_{1} \equiv k_{2} \equiv 0\right)$. We suppose the planes given by $x_{3}=$ constant. Then the foliations $\mathscr{G}$ corresponding to a completely integrable Pfaff differential system are determined by local distinguished submersions of the form $\varphi: U \rightarrow \mathrm{R}^{2}\left(U\right.$ open in $\left.\mathrm{R}^{3}\right)$, where $\varphi\left(x_{1}, x_{2}, x_{3}\right)=\left(g\left(x_{1}, x_{2}\right), x_{3}\right)$, with $g: U \rightarrow \mathrm{R}$ independent of $x_{3}$ and satisfying an eikonal equation of the form $|\mathrm{d} g|^{2}=1$. Local solutions to such an eikonal equation can be obtained by the method of characteristics and are determined by initial data on a smooth hypersurface. For any such solution $g$, the admissible function $\kappa: U \rightarrow \mathrm{R}$ is independent of $x_{3}$ and is given by

$$
\kappa=2\left(\frac{\partial g}{\partial x_{1}}\right)\left(\frac{\partial g}{\partial x_{2}}\right)\left(\frac{\partial^{2} g}{\partial x_{1} \partial x_{2}}\right)-\frac{\partial^{2} g}{\partial x_{1}^{2}}-\frac{\partial^{2} g}{\partial x_{2}^{2}} .
$$

(b) Co-axial cylinders $\left(k_{1} \neq 0, k_{2} \equiv 0\right)$. We introduce cylindrical coordinates by setting $\left(x_{1}, x_{2}, x_{3}\right)=(r \cos \psi, r \sin \psi, z)$. We suppose the cylinders are given by $r=$ constant. Then with respect to the frame $e_{1}=\partial / \partial z, e_{2}=\frac{1}{r} \frac{\partial}{\partial \theta}$, $e_{3}=\partial / \partial r$, solutions are given by $\alpha=\arctan (A r)$, where $A$ is a constant, which we allow to be both 0 and $\infty$ to include the cases $\alpha=0, \pi / 2$, respectively. The leaves of $\mathscr{G}$ are geodesic in the leaves of $\mathscr{F}$ and $\kappa$ is identically zero and is therefore the only admissible function. For $A \neq 0, \infty$, the leaves of $\mathscr{G}$ are helices that fill out the various cylinders. When $A=0$, they are lines parallel to the $x_{3}$-axis and when $A=\infty$, they are circles contained in planes perpendicular to the $x_{3}$-axis and with centres on this axis.

(c) Concentric spheres (clearly the 2-sphere does not admit a globally defined 1-dimensional subfoliation by the Hopf-Poincaré Theorem).

Let $\mathscr{F}$ be the foliation of $\mathrm{R}^{3} \backslash\{0\}$ given by concentric spheres $(s, \psi) \mapsto$ $r\left(\cos s, \sin s e^{i \psi}\right)$, for $r \in(0, \infty)$. Let $e_{1}=\frac{1}{r} \frac{\partial}{\partial s}, e_{2}=\frac{1}{r \sin s} \frac{\partial}{\partial \psi}, e_{3}=\frac{\partial}{\partial r}$ be the canonical adapted basis defined for $s \neq 0, \pi / 2$. Then $k_{1}=k_{2} \equiv k=-1 / r$ and $\omega^{12}=\frac{\cot s}{r} \omega^{2}$. Noting that $\left(\nabla_{V} \theta\right) \circ \mathscr{N}=\sin y \cos y\left(k_{1}-k_{2}\right) \omega^{3}$ vanishes, the corresponding Pfaff differential system takes the form

$$
\mathrm{d} y+\frac{\cot s}{r} \omega^{2}+\kappa V^{b}=0 .
$$


Let $\alpha$ be a solution. By umbilicity, from $(10), e_{3}(\kappa)+(\kappa / r)=0$, which integrates to give $\kappa=A(s, \psi) / r$ for some function $A$ of $s$ and $\psi$ only. From (15), $\partial \alpha / \partial r \equiv 0$ and

$$
\left\{\begin{array}{l}
\frac{\partial \alpha}{\partial s}=-A(s, \psi) \cos \alpha \\
\frac{\partial \alpha}{\partial \psi}=-\cos s-A(s, \psi) \sin s \sin \alpha
\end{array}\right.
$$

One solution is given by $\alpha \equiv \pi / 2$ in which case $A=-\cot s$. Otherwise eliminating $A$ (which is always almost everywhere non-zero), we obtain

$$
\tan \alpha \frac{\partial \alpha}{\partial s}-\frac{1}{\sin s} \frac{\partial \alpha}{\partial \psi}=\cot s .
$$

as a necessary and sufficient condition for $V=\cos \alpha e_{1}+\sin \alpha e_{2}$ to be tangent to a conformal foliation. Once more equation (16) can be solved by the method of characteristics.

For a solution $\alpha=\alpha(x)$ of (16), We set $A(s, \psi)=-\frac{1}{\cos \alpha} \frac{\partial \alpha}{\partial s}$. Then the admissible functions $\kappa$ are given by $\kappa=A(s, \psi) / r$.

A simple calculation shows that $\mathrm{d} W^{\mathrm{b}}=-\frac{1}{r} W^{\mathrm{b}} \wedge \omega^{3}$, so that $\mathrm{d}\left(W^{\mathrm{b}} / r\right)=0$ and locally (on a simply connected open set) $W^{\mathrm{b}} / r=\mathrm{d} g$ for some function $g$ satisfying $|\mathrm{d} g|^{2}=1 / r^{2}$. Now choose $f=\ln r$, so that the levels of $f$ are the leaves of $\mathscr{F}$. Then $|\mathrm{d} f|^{2}=1 / r^{2}$ and $\langle\mathrm{d} f, \mathrm{~d} g\rangle=0$, so that the pair $(f, g): U \subset \mathbf{R}^{3} \rightarrow \mathbf{R}^{2}$ determine a semi-conformal mapping with integral curves of $V$ as fibres.

(d) Totally geodesic non-parallel surfaces. We have a local product structure $\Pi \times(-\varepsilon, \varepsilon)$ where $\Pi$ is an open subset of a 2-dimensional Euclidean plane, and so coordinates $(u, v, s)$ with respect to which the Euclidean metric on $\mathrm{R}^{3}$ has the form

$$
g=\mathrm{d} u^{2}+\mathrm{d} v^{2}+f(u, v, s)^{2} \mathrm{~d} s^{2} .
$$

Here $u, v$ give Euclidean coordinates for each leaf of $\mathscr{F}$, and the three vector fields $e_{1}=\partial / \partial u, e_{2}=\partial / \partial v, e_{3}=(1 / f) \partial / \partial s$ form an orthogonal triple with $e_{3}$ normal to the leaves. In general $e_{3}$ is not geodesic, but satisfies $\left\langle\nabla_{e_{3}} e_{3}, e_{i}\right\rangle=$ $e_{i}(\ln (1 / f))$ for $i=1,2$. Since $\left(\mathscr{L}_{e_{i}} g\right)\left(e_{3}, e_{3}\right)=-2 g\left(\nabla_{e_{3}} e_{3}, e_{i}\right)=e_{i}\left(\ln f^{2}\right)$, $(i=1,2)$, we see that the divergence $a$ is determined by $a\left(e_{i}\right)=e_{i}\left(\ln f^{2}\right)$, $(i=1,2)$. It is easily checked that the form $\omega^{12}$ vanishes identically. Also, by total geodecity of the leaves, $\mathscr{N}\left(\nabla_{V} W\right)=0$, so that the Pfaff differential system (6) becomes

$$
\Omega_{\kappa} \equiv \mathrm{d} y-\frac{a(V)}{2} \theta+\kappa V^{b}=0 .
$$


Any solution $\alpha=\alpha(u, v, s)$ satisfies $\partial \alpha / \partial s=0$ and so is constant in normal directions. Set $a_{1}=a\left(e_{1}\right), a_{2}=a\left(e_{2}\right)$. Then equations (10) for complete integrability are equivalent to the following system:

$$
\begin{array}{r}
\partial a_{1} / \partial s=\partial a_{2} / \partial s=\partial \kappa / \partial s=0 \\
a_{1}^{2}+2 \frac{\partial a_{1}}{\partial u}=a_{2}^{2}+2 \frac{\partial a_{2}}{\partial v}=0 \\
a_{1} a_{2}+\frac{\partial a_{1}}{\partial v}+\frac{\partial a_{2}}{\partial u}=0 \\
\kappa a_{2}-2 \frac{\partial \kappa}{\partial v}=\kappa a_{1}-2 \frac{\partial \kappa}{\partial u}=0
\end{array}
$$

Then equations (17) integrate to give $a_{1}=2 /(u+\xi(v)), a_{2}=2 /(v+\eta(u))$, for some functions $\xi=\xi(v), \eta=\eta(u)$. Differentiating the first equation of (19) with respect to $u$ and the second with respect to $v$ and subtracting yields

$$
\kappa\left(\frac{\partial a_{1}}{\partial v}-\frac{\partial a_{2}}{\partial u}\right)=0,
$$

so that either $\kappa=0$ or $\partial a_{1} / \partial v=\partial a_{2} / \partial u$. In the latter case $\partial \xi / \partial v=\partial \eta / \partial u$ and $\xi=p v+q, \eta=p u+r$ for constants $p, q, r$. Equation (18) now gives $1-p^{2}=0$ and $2 q r-p\left(q^{2}+r^{2}\right)=0$ having solutions $(p, q, r)=$ $(1, q, q),(-1, q,-q)$. Then

$$
a_{1}=\frac{2}{u+v+q}, \quad a_{2}=\frac{2}{u+v+q}
$$

or

$$
a_{1}=\frac{2}{u-v+q}, \quad a_{2}=\frac{-2}{u-v+q}
$$

respectively. In this case both functions $a_{1}, a_{2}$ approach infinity as $(u, v)$ approaches the line $u \pm v+q=0$, showing that this line represents a common envelope for the planes and all planes must pass through this line. From (20), (21) and (19), we deduce that $\kappa=A(u+v+q)$ or $\kappa=A(u-v+q)$, respectively, for an arbitrary non-zero constant $A$.

If on the other hand $\kappa=0$, then once more using equation (18), we deduce that $\xi, \eta$ are both linear of the form $\xi=p r+q, \eta=\frac{1}{p} u+r$, where $p$ is an arbitrary non-zero constant and $r=q / p$. This gives

$$
a_{1}=\frac{2}{u+p v+q}, \quad a_{2}=\frac{2 p}{u+p v+q} .
$$


Once more the line $u+p v+q=0$ gives a common envelope through which all the planes pass.

For example, let us return to standard coordinates $\left(x_{1}, x_{2}, x_{3}\right)$ and consider the foliation $\mathscr{F}$ of $\mathrm{R}^{3} \backslash\left\{x_{3}-\right.$ axis $\}$ by half-planes which extend to the $x_{3}$-axis. Then for any $z \in \mathrm{R}$, the family of lines passing through the point $(0,0, z)$ determines a conformal subfoliation $\mathscr{G}$ of $\mathscr{F}$. Choose an arbitrary point $x_{0} \in$ $\mathrm{R}^{3} \backslash\left\{x_{3}-\right.$ axis $\}$; as $z$ increases from $-\infty$ to $+\infty$ we obtain all directions $V$ at $x_{0}$ except the directions parallel to the $x_{3}$-axis. But this is a limiting case as $z \rightarrow \pm \infty$.

This completes the proof of the theorem.

\section{Conformal flags in dimension four}

Let $M^{m}$ be an even dimensional manifold endowed with an almost complex structure $J$ and a compatible Riemannian metric $g$ i.e. $g(J X, J Y)=g(X, Y)$ for all tangent vectors $X, Y$ on $M$. The triplet $(M, g, J)$ defines an almost Hermitian structure. Associated to any almost Hermitian structure, there exists a non-degenerate 2-form $A$ defined by $A(X, Y)=g(X, J Y)$. In dimension 4, we can naturally associate a 1 -form $\beta$, called the Lee form [8], defined by

$$
\mathrm{d} A+\beta \wedge A=0
$$

The 1-form $\beta$ is identically zero if and only if the manifold is Kähler. We call points where $\beta$ vanishes, Kähler points.

Let $\left(M^{4}, g, J\right)$ be an almost Hermitian 4-manifold and let $\beta$ be the associated Lee form. Let $\mathscr{F}$ be an oriented 2-dimensional conformal foliation of $M$ which is invariant under $J$, i.e. $J: T \mathscr{F} \rightarrow T \mathscr{F}$. Note that, given a 2dimensional oriented foliation $\mathscr{F}$ of an oriented Riemannian 4-manifold $M^{4}$, we can always define such a $J$ by setting $J=\left(J^{T}, J^{N}\right)$, where $J^{T}$ is rotation by $+\pi / 2$ in $T \mathscr{F}$ and $J^{N}$ is rotation by $+\pi / 2$ in $N \mathscr{F}$.

Let $\left\{e_{1}, e_{2}, e_{3}, e_{4}\right\}$ be a local frame defined on an open set $U \subset M$ such that $\left\{e_{1}, e_{2}\right\}$ spans $T \mathscr{F}$. Let $\left\{\omega^{1}, \ldots, \omega^{4}\right\}$ be the dual frame. We suppose the frame positively oriented, so that $J e_{1}=e_{2}$ and $J e_{3}=e_{4}$. On $U$, we can write $A=\omega^{1} \wedge \omega^{2}+\omega^{3} \wedge \omega^{4}$.

LEMMA 4.1. The foliation $\mathscr{F}$ is minimal if and only if $\beta^{\sharp} \in T \mathscr{F}$.

Proof. For any $X \in N \mathscr{F}$, we have $\mathrm{d} A\left(e_{1}, e_{2}, X\right)+\beta(X)=0$. Also $\mathrm{d} A\left(e_{1}, e_{2}, X\right)=\sum_{i=1,2} \mathrm{~d} \omega^{i}\left(e_{i}, X\right)$, where $\mathrm{d} \omega^{i}\left(e_{i}, X\right)=-g\left(e_{i},\left[e_{i}, X\right]\right)=$ $g\left(\nabla_{e_{i}} e_{i}, X\right)$, so that

$$
\mathrm{d} A\left(e_{1}, e_{2}, X\right)=g\left(X, \sum_{i=1,2} \nabla_{e_{i}} e_{i}\right)
$$


where $\nabla$ denotes the Levi-Civita connection of $g$. But the right-hand-side represents twice the mean curvature of the leaves of $\mathscr{F}$ in the direction of $X$ and the result follows.

In what follows, we suppose that $\mathscr{F}$ is a 2-dimensional minimal conformal foliation of an almost Hermitian 4-manifold $\left(M^{4}, g, J\right)$ which is invariant under $J$. We suppose further that there are no Kähler points. We choose an adapted orthonormal frame field $\left\{e_{1}, e_{2}, e_{3}, e_{4}\right\}$ by setting $e_{1}=\beta^{\sharp} /|\beta|$ and $e_{2}=J \beta^{\sharp} /|\beta|$, so that $\left\{e_{1}, e_{2}\right\}$ spans $T \mathscr{F}$.

LeMma 4.2. The connection form $\omega^{12}$ satisfies

$$
\omega^{12}\left(e_{1}\right)=e_{2}\left(\ln \frac{|\beta|}{\lambda^{2}}\right),
$$

where $\lambda$ is any local dilation for $\mathscr{F}$.

If in addition the co-differential $\delta \beta$ defined by $\delta \beta=-\left(\nabla_{e_{i}} \beta\right)\left(e_{i}\right)$ vanishes, we also have

$$
\omega^{12}\left(e_{2}\right)=-e_{1}\left(\ln \frac{|\beta|}{\lambda^{2}}\right) .
$$

Proof. We may choose the normal vector fields $e_{3}, e_{4}$ so that $e_{3} / \lambda, e_{4} / \lambda$ are basic with respect to a distinguished submersion. In this case

$$
\mathcal{N}\left[e_{i}, e_{r}\right]=e_{i}(\ln \lambda) e_{r} \quad i=1,2 ; \quad r=3,4 .
$$

From (22), we have $\mathrm{d} A\left(e_{2}, e_{3}, e_{4}\right)=0$, which, by applying (23), is equivalent to

$$
e_{2}\left(\ln \lambda^{2}\right)+g\left(e_{1},\left[e_{3}, e_{4}\right]\right)=0 .
$$

Differentiating (22) gives $\mathrm{d} \beta \wedge A=0$, which is equivalent to

$$
-e_{2}(\ln |\beta|)-g\left(e_{1},\left[e_{3}, e_{4}\right]\right)+\omega^{12}\left(e_{1}\right)=0 .
$$

Combining these two expressions yields the first relation.

In the case when $\delta \beta=0$, we note that, by applying (23) once more,

$$
\left(\nabla_{e_{i}} \beta\right)\left(e_{i}\right)=e_{1}\left(\beta\left(e_{1}\right)\right)-\beta\left(\nabla_{e_{i}} e_{i}\right)=e_{1}(|\beta|)+|\beta| \omega^{12}\left(e_{2}\right)-|\beta| e_{1}\left(\ln \lambda^{2}\right) .
$$

The formula follows.

Let $\mathscr{F}$ be an arbitrary $k$-dimensional foliation of a Riemannian manifold $\left(M^{m}, g\right)$. Let $f: M \rightarrow \mathrm{R}$ be a smooth function. We define the tangent Laplacian to $\mathscr{F}$ at each $x \in M$ by

$$
\Delta^{\mathscr{F}} f=\Delta^{F}\left(\left.f\right|_{F}\right),
$$


where $F$ is the leaf of $\mathscr{F}$ passing through $x$ and $\Delta^{F}$ is the Laplacian on $\left(F,\left.g\right|_{F}\right)$.

LEMma 4.3. If $\mathscr{F}$ is minimal then $\Delta^{\mathscr{F}} f=\operatorname{trace}_{T \mathscr{F}} \nabla \mathrm{d} f$.

Proof. By definition, summing over $i=1, \ldots k$,

$$
\begin{aligned}
\Delta^{\mathscr{F}} f & =e_{i}\left(e_{i}(f)\right)-\mathrm{d} f\left(\mathscr{T} \nabla_{e_{i}} e_{i}\right) \\
& =\nabla \mathrm{d} f\left(e_{i}, e_{i}\right)+\mathrm{d} f\left(\nabla_{e_{i}} e_{i}\right)-\mathrm{d} f\left(\mathscr{T} \nabla_{e_{i}} e_{i}\right) \\
& =\nabla \mathrm{d} f\left(e_{i}, e_{i}\right)+\mathrm{d} f\left(\mathcal{N} \nabla_{e_{i}} e_{i}\right) \\
& =\nabla \mathrm{d} f\left(e_{i}, e_{i}\right),
\end{aligned}
$$

by the minimality of the leaves.

We return to the case when $\mathscr{F}$ is a 2-dimensional minimal conformal foliation in an almost Hermitian 4-manifold invariant under the almost complex structure $J$. We recall the notation defined in Section 2; thus, if $\mathscr{G}$ is a 1dimensional oriented subfoliation of $\mathscr{F}$, then $V=\cos \alpha e_{1}+\sin \alpha e_{2}$ denotes its unit tangent vector field. We set $W=J V$.

Proposition 4.4. Suppose $\delta \beta=0$. If $\mathscr{G}$ is a 1-dimensional conformal subfoliation of $\mathscr{F}$, then the following fundamental equations are satisfied:

(i) $V(\alpha)+W\left(\ln \left(|\beta| / \lambda^{2}\right)\right)+\kappa=0$;

(ii) $W(\alpha)-V\left(\ln \left(|\beta| / \lambda^{2}\right)\right)+V(\ln \lambda)=0$;

(iii) $\Delta^{\mathscr{F}}\left(\ln \left(|\beta| / \lambda^{2}\right)\right)=K-\kappa^{2}-(V(\ln \lambda))^{2}+[V, W](\alpha)$, where $\kappa$ is the geodesic curvature of the leaves of $\mathscr{G}$ in $\mathscr{F}$ and $\lambda$ is any local dilation.

Proof. The first two equations follow from Corollary 2.3 and Lemma 4.2. The third equation is obtained after differentiating (ii) with respect to $V$, (i) with respect to $W$, subtracting and then applying Lemma 4.3 together with Proposition 2.7.

COROLlary 4.5. If the function $\alpha$ is constant and $K \leq 0$, then the function $\ln \left(|\beta| / \lambda^{2}\right)$ is a subharmonic function on each leaf, in particular it can have no local minimum unless it is constant. If the leaves are compact $(K \equiv 0)$ then $\ln \left(|\beta| / \lambda^{2}\right)$ is constant along each leaf. If further $\mathscr{F}$ is Riemannian, then $|\beta|$ is constant along each leaf.

Remark 4.6. If we allow Kähler points, then if $|\beta|$ is constant along each leaf, either a leaf consists entirely of Kähler points, or it consists of non-Kähler points.

We wish to find good candidates, i.e. vector fields which generate a 1dimensional conformal subfoliation. In the absence of Kähler points, two natural possibilities are (i) $V=\beta^{\sharp} /|\beta|$, and (ii) $V=J \beta^{\sharp} /|\beta|$. 
Lemma 4.7. (i) The vector field $V=e_{1}=\beta^{\sharp} /|\beta|$ is tangent to a conformal foliation if and only if $e_{1}(\ln \lambda)=-\omega^{12}\left(e_{2}\right)$.

(ii) The vector field $V=e_{2}=J \beta^{\sharp} /|\beta|$ is tangent to a conformal foliation if and only if

$$
e_{2}\left(\ln \frac{|\beta|}{\lambda^{3}}\right)=0, \quad \mathrm{~d} \beta\left(e_{2}, e_{3}\right)=0, \mathrm{~d} \beta\left(e_{2}, e_{4}\right)=0 .
$$

In case (i), the geodesic curvature of the leaves in $\mathscr{F}$ is given by $\kappa=$ $-e_{2}\left(\ln \left(|\beta| / \lambda^{2}\right)\right)$ and in case (ii), by $\kappa=-\omega^{12}\left(e_{2}\right)$.

Proof. We apply Corollary 2.3, choosing the function $\kappa$ appropriately.

(i) We note that $\omega^{12} \circ \mathcal{N}+\left(\mathcal{N} \nabla_{e_{1}} e_{2}\right)^{\mathrm{b}}$ vanishes. Indeed, for $X \in N \mathscr{F}$,

$$
\omega^{12}(X)+g\left(\nabla_{e_{1}} e_{2}, X\right)=-g\left(e_{1}, \nabla_{e_{2}} X+\nabla_{X} e_{2}\right)=\left(\mathscr{L}_{e_{1}} g\right)\left(e_{2}, X\right)
$$

which vanishes by the assumption that $e_{1}$ be tangent to a conformal foliation. The expression for $\kappa$ follows from Lemma 4.2.

(ii) Evaluating the left-hand-side of (4) on normal vectors, say $e_{3}$, we find,

$$
\begin{aligned}
\omega^{12}\left(e_{3}\right)-\left(\mathcal{N} \nabla_{e_{2}} e_{1}\right)^{\mathrm{b}}\left(e_{3}\right) & =g\left(\nabla_{e_{3}} e_{1}, e_{2}\right)+g\left(e_{1}, \nabla_{e_{2}} e_{3}\right) \\
& =g\left(e_{1},\left[e_{2}, e_{3}\right]\right) \\
& =-\frac{1}{|\beta|} \mathrm{d} \beta\left(e_{2}, e_{3}\right) .
\end{aligned}
$$

The equations follow by equating the remaining coefficients to zero.

Proposition 4.8. (i) If $\delta \beta=0$, then $\beta^{\sharp}$ is tangent to a conformal foliation if and only if $e_{1}\left(\ln \left(|\beta| / \lambda^{3}\right)\right)=0$. In particular, if $e_{1}\left(\lambda^{2}\right)=0$ (which is the case if $\mathscr{F}$ is Riemannian), this is equivalent to $g\left(\nabla|\beta|, \beta^{\sharp}\right)=0$.

(ii) (a) If $\beta \wedge \mathrm{d} \beta=0$ (the Lee form is integrable), then $J \beta^{\sharp}$ is tangent to a conformal foliation if and only if $e_{2}(|\beta|)=0$, i.e. $g\left(\nabla|\beta|, J \beta^{\sharp}\right)=0$.

(b) If $e_{2}\left(\lambda^{2}\right)=0$ (which is the case if $\mathscr{F}$ is Riemannian) and $J \beta^{\sharp}$ is tangent to a conformal foliation, then $\beta \wedge \mathrm{d} \beta=0$, i.e. $\beta$ is integrable. In this case, necessarily, $g\left(\nabla|\beta|, J \beta^{\sharp}\right)=0$.

Proof. Part (i) follows from Lemmas 4.2 and 4.7(i).

For Part (ii), we note that $\beta \wedge \mathrm{d} \beta=0$ is equivalent to the vanishing of $\mathrm{d} \beta\left(e_{2}, e_{3}\right), \mathrm{d} \beta\left(e_{2}, e_{4}\right)$ and $\mathrm{d} \beta\left(e_{3}, e_{4}\right)$. But $\mathrm{d} \beta\left(e_{3}, e_{4}\right)=-\beta\left(\left[e_{3}, e_{4}\right]\right)=$ $|\beta| e_{2}\left(\ln \lambda^{2}\right)$ from (24). The result now follows from Lemma 4.7(ii).

By applying the above results, the existence of conformal flags can be used to deduce properties of almost Hermitian structures. In the following example, 
we construct a family of almost Hermitian (non-Kähler) structures on an open dense subset of $S^{4}$ with integrable Lee forms.

EXAMPLE 4.9 (Flags of conformal foliations in $S^{4}$ ). We consider $S^{4}$ parametrized in the form $\left(\cos s, \sin s\left(\cos t e^{i a}, \sin t e^{i b}\right)\right)$, where $s \in[0, \pi], t \in$ $[0, \pi / 2], a, b \in\left[0,2 \pi\right.$ [. We construct a semi-conformal map $\Phi_{k, l}: S^{4} \rightarrow S^{2}$ as the composition of two semi-conformal maps $\psi: S^{4} \rightarrow S^{3}$ and $\zeta_{k, l}: S^{3} \rightarrow$ $S^{2}$. The first map $\psi$ has the form $\psi(\cos s, \sin s x)=(\cos f(s), \sin f(s) H(x))$ where $x \in S^{3}$ and $H: S^{3} \rightarrow S^{2}$ is the Hopf fibration. The function $f(s)=$ $2 \arctan \left(\tan ^{2}(s / 2)\right)$ is chosen to render the map semi-conformal. The second $\operatorname{map} \zeta_{k, l}: S^{3} \rightarrow S^{2}$ is given by $\zeta_{k, l}\left(\cos u e^{\mathrm{i} \mu}, \sin u e^{\mathrm{i} v}\right)=(\cos \gamma(u), \sin \gamma(u)$ $\left.e^{\mathrm{i}(k \mu+l v)}\right)$, where $k, l$ are non-zero integers, $u=u(s, t), \mu=\mu(s, t)$ are given by a change of parameters, $v=a+b$ and where $\gamma$ is chosen to render $\zeta_{k, l}$ semi-conformal; this is equivalent to the equation

$$
\frac{\gamma^{\prime}}{\sin \gamma}=\sqrt{\frac{k^{2}}{\cos ^{2} u}+\frac{l^{2}}{\sin ^{2} u},}
$$

which can be solved explicitly in terms of elliptic functions [2].

Let $g_{S^{4}}$ denote the standard metric on $S^{4}$. In [3], the second author shows that with respect to the conformally Euclidean metric

$$
g=\frac{2}{\sqrt{\left(k^{2} \sin ^{2} 2 t+l^{2} \cos ^{2} 2 t\right)\left(\frac{\sin ^{4} s}{4}\right)+l^{2} \cos ^{2} s}} g_{S^{4}}
$$

the map $\Phi_{k, l}$ is harmonic and so a harmonic morphism. In particular, the regular fibres of $\Phi_{k, l}:\left(S^{4}, g\right) \rightarrow S^{2}$ form a conformal foliation by minimal surfaces of an open dense subset of $S^{4}$. On this subset we associate the almost Hermitian structure $J=J_{k, l}=\left(J^{T}, J^{N}\right)$. The corresponding Lee form $\beta$ is given by

$$
\beta=C(u, \mu)\left\{k \sin ^{2} u(\mathrm{~d} a+\mathrm{d} b)-l \cos ^{2} u \mathrm{~d} \mu\right\},
$$

where $C(u, \mu)=2 l \cos ^{2} u \sin ^{2} \mu /\left(l^{2} \cos ^{2} u+k^{2} \sin ^{2} u\right) \sin f$. It follows that $J \beta^{\sharp}$ is proportional to the vector field $\frac{\partial}{\partial a}-\frac{\partial}{\partial b}$, so that the fibres of $\psi$, which determine a conformal subfoliation of the regular fibres of $\Phi_{k, l}$, are generated by $J \beta^{\sharp}$. Since the dilation $\lambda$ of $\Phi_{k, l}$ is independent of $a$ and $b$, we see that $\left(J \beta^{\sharp}\right)\left(\lambda^{2}\right)$ vanishes. By Proposition 4.8, the almost Hermitian structure $J$ has integrable Lee form.

Finally, we describe an example where the corresponding Pfaff differential system (6) is completely integrable. 
Example 4.10. Consider $S^{3} \times S^{1}$ parametrized in the form $\left(\left(\cos s e^{i a}\right.\right.$, $\left.\left.\left.\sin s e^{i b}\right), e^{i c}\right)\right), s \in\left[0, \pi / 2\left[, a, b, c \in\left[0,2 \pi\left[\right.\right.\right.\right.$. Define $\Phi_{k, l}: S^{3} \times S^{1} \rightarrow S^{2}$ by

$$
\Phi_{k, l}\left(\left(\cos s e^{i a}, \sin s e^{i b}\right), e^{i a}\right)=\left(\cos \gamma(s), \sin \gamma(s) e^{i(k a+l b)}\right)
$$

where $k, l$ are non-zero integers and where $\gamma(s)$ solves the equation

$$
\frac{\gamma^{\prime}(s)}{\sin \gamma(s)}=\sqrt{\frac{k^{2}}{\cos ^{2} s}+\frac{l^{2}}{\sin ^{2} s}}
$$

The regular fibres of $\Phi_{k, l}$ form a conformal foliation $\mathscr{F}$ by surfaces of an open dense subset of $S^{3} \times S^{1}$. Let $\left\{e_{1}, e_{2}\right\}$ span $T \mathscr{F}$ where $e_{1}=r(t)\left(l \frac{\partial}{\partial a}-k \frac{\partial}{\partial b}\right)$ and $e_{2}=\frac{\partial}{\partial c}$, with $r(t)$ a nowhere vanishing function. Extend to an orthonormal frame $\left\{e_{1}, e_{2}, e_{3}, e_{4}\right\}$ on an open dense subset of $S^{3} \times S^{1}$. Let $\left\{\omega^{i}\right\}(i=1 \ldots 4)$ be the dual frame. Set $V=\cos \alpha e_{1}+\sin \alpha e_{2}$. Let $\lambda=\lambda(t)$ denote the dilation of $\Phi_{k, l}$, we clearly have $V(\lambda)=0$. For $\kappa=0$, the Pfaff differential system given by

$$
\Omega_{\kappa} \equiv \mathrm{d} y-\sin y \cos y \frac{r^{\prime}(t)}{r(t)} d t=0
$$

clearly satisfies $\Omega_{\kappa} \wedge \mathrm{d} \Omega_{\kappa}=0$, and so is completely integrable.

\section{REFERENCES}

1. Aubin, T., A Course in Differential Geometry, Grad. Stud. Math. 27 (2000).

2. Baird, P., and $\mathrm{Ou}, \mathrm{Y}-\mathrm{L} .$, Harmonic maps and morphisms from multilinear norm-preserving mappings, Internat. J. Math. 8 (1997), 187-211.

3. Burel, J-M., Applications et morphismes harmoniques à valeurs dans une surface, C. R. Acad. Sci. Paris Ser. I Math. 332 (2001), 441-446.

4. Cartan, E., Familles de surfaces isoparamétriques dans les espaces à courbure constante, Ann. Mat. Pura Appl. 17 (1938), 177-191.

5. Cordero, L. A., and Masa, X., Characteristic classes of subfoliations, Ann. Inst. Fourier, Grenoble 31 (1981), 61-86.

6. Feigin, B. L., Characteristic classes of flags of foliations, Functional Anal. Appl. 9 (1975), 312-317.

7. Hector, G., and Hirsch, U., Introduction to the Geometry of Foliations, Parts A and B, Aspects Math., 1981.

8. Lee, H. C., A kind of even dimensional differential geometry and its applications to exterior calculus, Amer. J. Math. 65 (1943), 433-438. 
9. Spivak, M., A Comprehensive Introduction to Differential Geometry, Vol. II, 2nd ed., Publish or Perish, Berkeley, California, 1979.

10. Vaisman, I., Conformal foliations, Kodai Math. J. 2 (1979), 26-37.

DÉPARTEMENT DE MATHÉMATIQUES

UNIVERSITÉ DE BRETAGNE OCCIDENTALE

6 AVENUE LE GORGEU

B.P. 452

29275 BREST CEDEX

FRANCE

E-mail: Paul.Baird@univ-brest.fr
CENTRE FOR MATHEMATICAL SCIENCES

LUND UNIVERSITY, BOX 118

S-22100 LUND

SWEDEN

E-mail: Jean-Marie.Burel@math.lu.se 